\title{
Estimation of Thermodynamic Properties of Binary Liquid Mixtures on the Basis of Statistical Mechanical Theories
}

\author{
J. D. Pandey, Prakash Chandra, Rupali Sethi and Vinay Sanguri* \\ Department of Chemistry, University of Allahabad, Allahabad, India \\ Email: drvsanguri@ rediffmail.com
}

\begin{abstract}
Thermodynamic properties of liquids and liquid mixtures play very important role in understanding the nature of molecular interactions occurring in the system. In the present work different thermodynamic properties of 15 pure liquids and 34 equimolar binary liquid mixtures of benzene, toluene, p-xylene, chlorobenzene and 1chloronaphthalene with linear and branched alkanes have been computed with the help of Flory's statistical theory (FST), Hard sphere equation of state (HSE) and Hole theory (HT) simultaneously. The calculated values are compared with the experimental findings collected from literature and quite satisfactory results are obtained.
\end{abstract}

Keywords: Flory statistical theory; hard sphere equation of state; hole theory; binary liquid mixtures.

\section{Introduction}

During the recent past it has been customary to utilize ultrasonic velocity data for the computation of a number of interesting and useful equilibrium and transport properties of liquids and liquid mixtures (Acosta et al., 2001; Dhasi et al., 1998; Ali \& Tiwari et al., 2000; Mosteriro et al., 2001; Ali \& Nain, 2002; Baluja, 2002; Ali \& Ahmad et al., 2000; Arce et al., 2000; Canosa et al., 2001). Since velocity and density measurements are quite simple and economical, such studies are useful in estimating these properties. Also, some of the properties which cannot be determined directly from the experimental method, they can be obtained from velocity and density data. On the other hand, several empirical and semi-empirical procedures (Nomoto, 1958; Van Dael, 1975; Jakobson, 1951; Schaffs, 1939; Jain et al., 1974; Kaulgud \& Patil, 1972; Acree (Jr), 1984; Reid et al., 1987; Rowlinson \& Swinton, 1982; Murrell \& Jenkins, 1982; Prausnitz et al., 1986; Eyring \& John, 1969; Pandey et al., 1997; Pandey et al., 1999; Pandey \& Vyas et al., 1999; Ali \& Nain, 2000; Ali \& Nain, 2002) have also been used to estimate theoretically the sound velocity. Due to the importance of ultrasonic velocity, various liquid state models have been employed successfully to correlate this property with other, and also a theoretical evaluation has been carried out. It would be interesting to mention some liquid state models e.g. Flory's statistical theory (FST) (Flory et al., 1964; Flory, 1965; Abe \& Flory, 1965; Bloomfeld \& Dewan, 1971; Dewan et al., 1971; Pandey \& Alec David, 1981; Pandey \& Sanguri, 2008; Pandey \& Sanguri, 2001; Pandey \& Sanguri et al., 2002; Pandey \& Sanguri et al., 2003; Dey \& Pandey et al., 2006), Hard sphere equation of state (HSE) (Percus \& Yevick, 1958; Lebowitz et al., 1965; Thiele, 1963; Lebowitz \& Frisch et al., 1961; Carnahan \& Starling, 1969; Frisch, 1964; Hoover \& Ree, 1964) and Hole theory (HT) (Pandey \& Sanguri et al., 2010; Pandey \& Sanguri et al., 2007; Pandey, Dwivedi \& Sanguri, 2008; Sanguri et al., 2008), that were utilized to deduce ultrasonic velocity and other thermodynamic properties of liquids and liquid mixtures (binary and higher orders). A comparative study of the applicability of various liquid state theories employed for the estimation of thermodynamic and other related properties has, so far, not been done. An entirely different procedure has been adopted in the present work. The present work has the following merits:

(i) To make a comparative study of Flory's statistical theory (FST), Hard sphere equation of state (HSE) and Hole theory (HT) on the basis of theoretically computed values of various thermodynamic properties of 15 pure liquids and 34 equimolar binary liquid mixtures.

(ii) An attempt has been made to evaluate ultrasonic velocities in the above mentioned systems from the experimental values of thermal expansivity $(\alpha)$, heat capacity at constant pressure $\left(\mathrm{C}_{\mathrm{P}}\right)$ and isothermal compressibility $\left(\beta_{\mathrm{T}}\right)$. These values of sonic velocities are treated as experimental values.

(iii) These experimentally deduced values of velocity are compared with the theoretical ones obtained from FST and HT.

(iv) Nine different physical properties (viz. $\beta_{\mathrm{T}}, \beta_{\mathrm{S}}, \alpha$, $\mathrm{C}_{\mathrm{P}}, \mathrm{C}_{\mathrm{V}}, \mathrm{P}_{\mathrm{i}}, \gamma, \Gamma$ and $\mathrm{u}$ ) are theoretically calculated by FST and HT and compared with the experimental findings.

(v) $\alpha$ and $\beta_{\mathrm{T}}$ are also calculated with the help of seven different HSE and compared with the experimental values.

(vi) Theoretical estimation of the density of equimolar binary mixtures has been done with the help of FST.

These studies are made by using the typical experimental data of Tardajos \& Patterson et al., 1986; 
Costas \& Patterson et al., 1988; Dominguez \& Patterson et al., 1993. Such types of data are very rare in the literature. They measured quite accurately and precisely molar volume $(\mathrm{V})$, thermal pressure coefficient $\left(\gamma_{\mathrm{P}}\right)$, thermal expansivity, isothermal compressibility and heat capacity at constant pressure of 15 pure liquids and their binary mixtures. In the present work, we are applying FST, HSE and HT for estimating the values of various thermodynamic properties of 15 pure liquids and 34 equimolar binary liquid mixtures of benzene, toluene, $\mathrm{p}$-xylene, chlorobenzene, and 1-chloronaphthalene with linear $\left(\mathrm{n}-\mathrm{C}_{6}, \mathrm{n}-\mathrm{C}_{8}, \mathrm{n}-\mathrm{C}_{10}, \mathrm{n}-\mathrm{C}_{12}\right.$, $\mathrm{n}-\mathrm{C}_{14} \& \mathrm{n}-\mathrm{C}_{16}$ ) and branched alkanes (br- $\mathrm{C}_{6}$, br- $\mathrm{C}_{8}$, br- $\mathrm{C}_{12}$ and $\mathrm{br}-\mathrm{C}_{16}$ ). As far as our knowledge is concerned, this is for the first time, when three very important liquid state theories (viz. FST, HSE and HT) are simultaneously employed for the estimation of physical properties of liquids and liquid mixtures.

\section{Theoretical}

Flory theory, HSE and HT have been recently (Flory et al., 1964; Flory, 1965; Abe \& Flory, 1965; Bloomfeld \& Dewan, 1971; Dewan et al., 1971; Pandey \& Alec David, 1981; Pandey \& Sanguri, 2008; Pandey \& Sanguri, 2001; Pandey \& Sanguri, 2002; Pandey \& Sanguri, 2003; Dey \& Pandey, 2006; Percus \& Yevick, 1958; Lebowitz et al., 1965; Thiele, 1963; Lebowitz \& Frisch et al., 1961; Carnahan \& Starling, 1969; Frisch, 1964; Hoover \& Ree, 1964; Pandey \& Sanguri et al., 2010; Pandey \& Sanguri et al., 2007; Pandey, Dwivedi \& Sanguri, 2008; Sanguri et al., 2008) utilized for computing various thermodynamic and transport properties of liquid mixtures. Various modifications of FST have been made and utilized successfully. The relevant equations are summarized here. The details of these equations are given in earlier papers (Pandey \& Sanguri, 2008; Pandey \& Sanguri, 2001; Pandey \& Sanguri et al., 2002; Pandey \& Sanguri et al., 2003; Dey $\&$ Pandey, 2006 et al., 2006).

The equilibrium properties of a liquid are strongly dependent on what may be loosely called its local structure, often expressed in terms such as packing density, free volume or, more exactly, in terms of the radial distribution function. Flory et al used a simple partition function proposed by Eyring \& Hirschfelder to get the reduced equation of state, which is based on vander Waals potential energy model. They assume the intermolecular energy to depend only on the volume, and a hard sphere repulsive potential is adopted for segments of the chain. The number of external degree of freedom is introduced as a parameter on the premise that the corresponding modes can be separated unambiguously from the internal degrees of freedom of molecules. From the knowledge of $\alpha, \beta_{\mathrm{T}}$ and V of pure components, the values of reduced and characteristic parameters for pure components can be obtained. With these parameters we can calculate various thermodynamic properties of liquids and liquid mixtures.
Thermal expansivity, density ( $\rho)$ isothermal compressibility, internal pressure $\left(\mathrm{P}_{\mathrm{i}}\right)$, heat capacity at constant pressure, adiabatic compressibility $\left(\beta_{\mathrm{s}}\right)$, ultrasonic velocity $(\mathrm{u})$, heat capacity ratio $(\gamma)$, heat capacity at constant volume $\left(\mathrm{C}_{\mathrm{V}}\right)$ and Gruneisen parameter $(\Gamma)$ by Flory theory are calculated with the help of following equations:

$$
\begin{aligned}
& \alpha=\frac{3\left(\tilde{V}^{1 / 3}-1\right)}{T\left(1-3\left(\tilde{V}^{1 / 3}-1\right)\right)} \\
& \rho=\frac{x_{1} M_{1}+x_{2} M_{2}}{\left(x_{1} V_{1}^{*}+x_{2} V_{2}^{*}\right)(\tilde{V})} \\
& \beta_{T}=\frac{\alpha T \tilde{V}^{2}}{P^{*}} \\
& P_{i}=\frac{\alpha T}{\beta_{T}} \\
& C_{P}=\left(x_{1} C_{P, 1}+x_{2} C_{P, 2}\right)+C_{P}^{E} \\
& \beta_{S}=\beta_{T}-\frac{\alpha^{2} T V}{C_{P}} \\
& C_{V}=\frac{\gamma-1}{\alpha T} \\
& u=\left(\frac{1}{\beta_{S} \rho}\right)^{1 / 2} \\
& \beta_{T} / \beta_{S} \\
& \Gamma=1
\end{aligned}
$$

The basic idea of the hole theory is to assume the existence of holes in liquid. A liquid can be treated as a solution of molecules and holes. The appearance of holes can be regarded as a particular case of hetrophase fluctuations. The holes can be treated as gas bubbles when these are large enough to accommodate a sufficient amount of vapours. Surface tension and internal pressure of pure liquid components are the only input data for estimating the hole radius, hole volume and hole creation energy in the present hole theory. From these three properties, it is possible to calculate various thermodynamic properties of liquids and liquid mixtures.

Isothermal compressibility, thermal expansivity and ultrasonic velocity of pure liquids by hole theory are calculated using the following equations: 


$$
\begin{aligned}
& \beta_{T}=\frac{a N V_{h}^{2} \exp \left(1-\frac{1}{a}\right) \exp \frac{\left(E_{h}+P V_{h}\right)}{k T}}{V k T\left[\exp \left(1-\frac{1}{a}\right) \exp \frac{\left(E_{h}+P V_{h}\right)}{k T}-1\right]^{2}} \\
& \alpha=\frac{1}{V k T^{2}} \frac{a N V_{h} 2 \exp \left(1-\frac{1}{a}\right)\left[\exp \frac{\left(E_{h}+P V_{h}\right)}{k T}\right]\left(E_{h}+P V_{h}\right)}{\left[\exp \left(1-\frac{1}{a}\right) \exp \frac{\left(E_{h}+P V_{h}\right)}{k T}-1\right]^{2}} \\
& u=\left(\frac{\gamma a R T}{M}\right)^{1 / 2} 2 \operatorname{Sinh}\left[\frac{1}{2}\left\{\exp \frac{\left(E_{h}+P V_{h}\right)}{k T}-1+\frac{1}{a}\right\}\right]
\end{aligned}
$$

The details of these equations and symbols are given in our earlier papers (Pandey \& Sanguri et al., 2010; Pandey \& Sanguri et al., 2007; Pandey, Dwivedi \& Sanguri, 2008; Sanguri et al., 2008).

The equations used to calculate isothermal compressibility, thermal expansivity and ultrasonic velocity of binary mixtures by hole theory are analogous to the above Eqs. (11), (12) and (13). Here we use molar volume of the mixture $\left(\mathrm{V}_{\text {mix }}\right)$, volume of a hole in the mixture $\left(\mathrm{V}_{\mathrm{h} \text { (mix) }}\right)$ and energy of creation of a hole in the mixture $\left(\mathrm{E}_{\mathrm{h} \text { (mix) }}\right)$ in place of $\mathrm{V}, \mathrm{V}_{\mathrm{h}} \& \mathrm{E}_{\mathrm{h}}$. The method of calculation of $\mathrm{V}_{\mathrm{h} \text { (mix) }}$ and $\mathrm{E}_{\mathrm{h} \text { (mix) }}$ is given in the literature (Pandey \& Sanguri et al., 2007). With the help of calculated values of $\beta_{\mathrm{T},} \alpha$ and $\mathrm{u}$ by hole theory, we have also calculate $\beta_{\mathrm{S}}, \mathrm{C}_{\mathrm{P},} \gamma$, $\mathrm{C}_{\mathrm{V}}$ using Eqs. (6), (7), (8), (9) and (10) respectively.

The equation of state proposed by vander Waals accounts in a remarkable manner for most essential features of fluid behaviour, it fails to give the accurate representation of experimental thermodynamic data required in practice. Therefore, many modifications of the equation have been proposed, which generally involve the introduction of a dependence of the parameter ' $\mathbf{a}$ ' and ' $\mathbf{b}$ ' on temperature and or density. With the help of virial equation of state and hard sphere potential, hard sphere equation of state is obtained. Various theoretical approaches have been suggested by different workers for solving the hard sphere problem at higher densities. Thermal expansion coefficient of pure liquids and binary liquid mixtures is also calculated by using seven different hard sphere models (viz. Thiele-Lebowitz, Thiele, Carnahan-Starling, Guggenheim, Scaled-particle theory, Henderson and Hoover-Ree model). The details about the equations used to calculate $\alpha$ are given in the literature (Pandey \& Sanguri et al., 2002). Similarly, isothermal compressibility of pure liquids and binary liquid mixtures are also calculated by aforesaid seven hard-sphere models. The equations required for computing $\beta_{\mathrm{T}}$ have been obtained from the literature (Pandey, Dubey et al., 1994). The packing fraction ' $y$ ', which is required for the calculation of $\alpha$ and $\beta_{\mathrm{T}}$ by different hard sphere models, has been obtained by the method suggested earlier (Pandey \& Sanguri et al., 2002).

\section{Results and Discussion}

The experimental values of $\mathrm{V}, \alpha, \beta_{\mathrm{T}}, \mathrm{C}_{\mathrm{p}}$ and $\gamma_{\mathrm{p}}$ at 298.15 $\mathrm{K}$ for fifteen pure liquids (chlorobenzene, 1chloronaphthalene, n- $\mathrm{C}_{6}, \mathrm{n}-\mathrm{C}_{8}, \mathrm{n}-\mathrm{C}_{10}, \mathrm{n}-\mathrm{C}_{12}, \mathrm{n}-\mathrm{C}_{14} \& \mathrm{n}-\mathrm{C}_{16}$, br- $\mathrm{C}_{6}$, br- $\mathrm{C}_{8}$, br- $\mathrm{C}_{12}$, br- $\mathrm{C}_{16}$, benzene, toluene and p-xylene) were collected from the papers of Tardajos \& Patterson et al., 1986; Costas \& Patterson et al., 1988; Dominguez \& Patterson et al., 1993. They have also reported the experimental values of these thermodynamic quantities for thirty-four equimolar binary mixtures of 1chloronaphthalene, chlorobenzene, benzene, toluene and pxylene with normal and branched chain alkanes mentioned above.

All the parameters of pure components are listed in Table 1. The necessary data needed, for the calculation, have been taken from different sources (Tardajos \& Patterson et al., 1986; Costas \& Patterson et al., 1988; Dominguez \& Patterson et al., 1993; CRC, 1997). Table 2 enlists the percentage deviations of different properties of pure components using hole theory and Flory theory. In Table 3, percentage deviations of different properties of binary mixtures calculated through Flory theory, have been presented. In the present work only percentage deviations of different properties have been reported. The computed values of the desired thermodynamic properties of pure components and binary liquid mixtures can be obtained from the authors on request.

For the comparison of theoretically computed values with the observed values under consideration, the experimental values of liquid mixtures were taken from different sources (Tardajos \& Patterson et al., 1986; Costas \& Patterson et al., 1988; Dominguez \& Patterson et al., 1993). The experimental values of $\rho, \beta_{s}, \gamma, C_{v}, P_{i}$ and $u$ of binary liquid mixtures were obtained by the following equations:

$$
\begin{aligned}
& \beta_{S_{\exp }}=\frac{1}{u_{\exp \rho_{\exp }}^{2}} \\
& C_{P_{(\exp )}}=\frac{\alpha^{2} \exp T V_{\exp }}{\beta_{T_{(\exp )}}-\beta_{S_{(\exp )}}} \\
& \gamma_{\exp }=\left(\frac{\beta_{T}}{\beta_{S}}\right) \\
& C_{V}=\frac{C_{P_{(\exp )}}}{\gamma_{\exp }} \\
& \Gamma_{\exp }=\frac{\gamma_{\exp }-1}{\alpha_{\exp } T} \\
& \text { and } P_{i}=\frac{\alpha_{\exp } T}{\beta_{T_{(\exp )}}}
\end{aligned}
$$


Table 1. Different properties of pure components at $298.15 \mathrm{~K}$

\begin{tabular}{|c|c|c|c|c|c|c|c|c|c|c|c|}
\hline Component & $V$ & $\begin{array}{c}\alpha \times 10^{3} \\
\mathrm{~K}^{-1}\end{array}$ & $\tilde{V}$ & $V^{*}$ & $\tilde{T}$ & $T^{*}$ & $\begin{array}{c}\beta_{\mathrm{T}} \times 10^{12} \\
\mathrm{~cm}^{2} \text { dyne }\end{array}$ & $\begin{array}{c}P^{*} \\
\mathrm{~J} \mathrm{~cm}^{-3}\end{array}$ & M & $\rho$ & $\begin{array}{c}C_{P} \\
\mathrm{JK}^{-1} \mathrm{~mol}^{-1}\end{array}$ \\
\hline chlorobenzene & 102.24 & 0.982 & 1.2440 & 82.1863 & 0.0564 & 5284.012 & 75.50 & 600.13 & 112.56 & 1.1009 & 153.78 \\
\hline 1-chloronaphthalene & 136.77 & 0.702 & 1.1833 & 115.5881 & 0.0461 & 6467.786 & 48.80 & 600.49 & 162.62 & 1.1890 & 212.60 \\
\hline$n-C_{6}$ & 131.563 & 1.381 & 1.3209 & 99.5987 & 0.0671 & 4444.85 & 166.90 & 430.46 & 86.18 & 0.6550 & 197.67 \\
\hline$n-C_{8}$ & 163.526 & 1.160 & 1.2796 & 127.7928 & 0.0617 & 4835.431 & 128.20 & 441.74 & 114.23 & 0.6985 & 254.07 \\
\hline$n-C_{10}$ & 195.92 & 1.041 & 1.2561 & 155.9808 & 0.0583 & 5117.714 & 109.40 & 447.59 & 142.28 & 0.7262 & 315.32 \\
\hline$n-C_{12}$ & 228.578 & 0.978 & 1.2432 & 183.8661 & 0.0563 & 5296.037 & 98.80 & 456.12 & 170.34 & 0.7452 & 376.09 \\
\hline$n-C_{14}$ & 261.339 & 0.931 & 1.2334 & 211.8878 & 0.0548 & 5445.317 & 91.00 & 464.02 & 198.39 & 0.7591 & 438.01 \\
\hline$n-C_{16}$ & 294.052 & 0.902 & 1.2273 & 239.6004 & 0.0538 & 5545.426 & 85.70 & 472.64 & 226.25 & 0.7694 & 500.21 \\
\hline $\mathrm{br}-\mathrm{C}_{6}$ & 133.697 & 1.460 & 1.3350 & 100.1495 & 0.0688 & 4335.187 & 198.20 & 391.41 & 86.18 & 0.6446 & 191.88 \\
\hline $\mathrm{br}-\mathrm{C}_{8}$ & 166.056 & 1.192 & 1.2858 & 129.1468 & 0.0625 & 4769.492 & 151.20 & 388.60 & 114.23 & 0.6879 & 242.49 \\
\hline $\mathrm{br}-\mathrm{C}_{12}$ & 229.621 & 0.977 & 1.2430 & 184.7358 & 0.0563 & 5299.059 & 113.80 & 395.47 & 170.34 & 0.7418 & 350.98 \\
\hline $\mathrm{br}-\mathrm{C}_{16}$ & 289.84 & 0.854 & 1.2170 & 238.1624 & 0.0521 & 5726.497 & 92.90 & 405.93 & 226.25 & 0.7806 & 458.80 \\
\hline benzene & 89.408 & 1.218 & 1.2908 & 69.2677 & 0.0632 & 4718.574 & 96.60 & 626.32 & 78.12 & 0.8737 & 136.67 \\
\hline toluene & 106.875 & 1.086 & 1.2651 & 84.4810 & 0.0596 & 5003.421 & 91.40 & 566.96 & 92.15 & 0.8622 & 158.67 \\
\hline p-xylene & 123.923 & 1.019 & 1.2516 & 99.0126 & 0.0576 & 5177.397 & 84.40 & 563.88 & 106.17 & 0.8567 & 183.65 \\
\hline
\end{tabular}

Table 2. Percentage deviation of different properties of pure components using Hole theory and Flory theory.

\begin{tabular}{|c|c|c|c|c|c|c|}
\hline Component & & $\% \beta_{\mathrm{T}}$ & $\% \beta_{\mathrm{s}}$ & $\% \alpha$ & $\% C_{p}$ & $\% u$ \\
\hline & theory) & \multicolumn{5}{|c|}{ (Hole theory) } \\
\hline chlorobenzene & 8.69 & -11.81 & -8.51 & -11.87 & 47.63 & 15.96 \\
\hline 1-chloronaphthalene & 9.01 & --- & --- & --- & --- & --- \\
\hline $\mathrm{n}-\mathrm{C}_{6}$ & -5.93 & 7.19 & 9.03 & 7.11 & 49.88 & 6.74 \\
\hline $\mathrm{n}-\mathrm{C}_{8}$ & -4.94 & -5.66 & -4.31 & -5.74 & 34.86 & 11.54 \\
\hline $\mathrm{n}-\mathrm{C}_{10}$ & -4.47 & -5.59 & -4.73 & -5.67 & 26.41 & 10.88 \\
\hline$n-C_{12}$ & -3.91 & -2.04 & -1.56 & -2.11 & 18.92 & 9.09 \\
\hline $\mathrm{n}-\mathrm{C}_{14}$ & -3.45 & -17.11 & -16.86 & -17.18 & -4.67 & 14.97 \\
\hline $\mathrm{n}-\mathrm{C}_{16}$ & -2.98 & -10.21 & -10.21 & -10.28 & -10.47 & 12.30 \\
\hline $\mathrm{br}-\mathrm{C}_{6}$ & -16.63 & 13.39 & 15.24 & 13.31 & 55.26 & 3.19 \\
\hline $\mathrm{br}-\mathrm{C}_{8}$ & -6.81 & -5.64 & -4.01 & -5.73 & 40.78 & 11.00 \\
\hline $\mathrm{br}-\mathrm{C}_{12}$ & -5.56 & --- & --- & --- & --- & --- \\
\hline $\mathrm{br}-\mathrm{C}_{16}$ & -4.28 & --- & --- & --- & --- & --- \\
\hline benzene & 6.78 & -4.63 & -1.30 & -4.69 & 48.42 & 15.76 \\
\hline toluene & 4.24 & -11.36 & -8.34 & -11.43 & 44.86 & 16.30 \\
\hline p-xylene & 3.84 & -16.25 & -13.80 & -16.31 & 36.37 & 17.66 \\
\hline
\end{tabular}


Table 3. Percentage deviation of different properties of binary mixtures calculated through Flory theory

\begin{tabular}{|c|c|c|c|c|c|c|c|c|c|c|c|}
\hline Components & $\mathrm{x}_{1}$ & $\% \beta_{\mathrm{T}}$ & $\% \beta_{\mathrm{S}}$ & $\% \alpha$ & $\% C_{p}$ & $\% \mathrm{P}_{\mathrm{i}}$ & $\% \gamma$ & $\% \rho$ & $\%$ u & $\% \Gamma$ & $\% C_{V}$ \\
\hline benzene $\left(x_{1}\right)+n-C_{6}\left(x_{2}\right)$ & 0.5000 & 3.26 & 3.72 & -0.16 & -1.79 & -3.53 & -0.48 & -0.44 & -1.69 & -1.75 & -1.30 \\
\hline benzene $\left(x_{1}\right)+n-C_{8}\left(x_{2}\right)$ & 0.5000 & 2.64 & 3.57 & -0.83 & -0.70 & -3.57 & -0.97 & -0.46 & -1.60 & -3.37 & 0.27 \\
\hline benzene $\left(x_{1}\right)+n-C_{10}\left(x_{2}\right)$ & 0.5000 & 2.61 & 3.09 & -0.63 & -1.72 & -3.32 & -0.49 & -0.42 & -1.37 & -1.66 & -1.22 \\
\hline benzene $\left(x_{1}\right)+n-C_{12}\left(x_{2}\right)$ & 0.5000 & 2.05 & 2.84 & -1.24 & -0.97 & -3.35 & -0.82 & -0.40 & -1.25 & -2.80 & -0.15 \\
\hline benzene $\left(x_{1}\right)+n-C_{14(}\left(x_{2}\right)$ & 0.5000 & 2.01 & & -2.05 & & -4.15 & & -0.38 & & & \\
\hline toluene $\left(x_{1}\right)+n-C_{6}\left(x_{2}\right)$ & 0.5000 & 2.40 & 2.49 & 0.65 & -0.60 & -1.80 & -0.09 & -0.25 & -1.14 & -1.03 & -0.51 \\
\hline toluene $\left(x_{1}\right)+n-C_{8}\left(x_{2}\right)$ & 0.5000 & 2.59 & 3.23 & 0.08 & 0.13 & -2.57 & -0.67 & -0.23 & -1.54 & -3.16 & 0.80 \\
\hline toluene $\left(x_{1}\right)+n-C_{10}\left(x_{2}\right)$ & 0.5000 & 1.74 & 2.11 & -0.21 & -0.51 & -1.98 & -0.38 & -0.21 & -0.97 & -1.64 & -0.12 \\
\hline toluene $\left(x_{1}\right)+n-C_{12}\left(x_{2}\right)$ & 0.5000 & 1.77 & 2.21 & -0.51 & -0.84 & -2.33 & -0.44 & -0.20 & -1.02 & -1.73 & -0.39 \\
\hline toluene $\left(x_{1}\right)+n-C_{14}\left(x_{2}\right)$ & 0.5000 & 1.43 & & -0.86 & & -2.32 & & -0.13 & & & \\
\hline$p$-xylene $\left(x_{1}\right)+n-C_{6}\left(x_{2}\right)$ & 0.5000 & 4.91 & 5.84 & 0.76 & -0.11 & -4.36 & -0.98 & -0.16 & -2.97 & -5.11 & 0.87 \\
\hline$p$-xylene $\left(x_{1}\right)+n-C_{16}\left(x_{2}\right)$ & 0.5000 & 2.60 & 3.42 & -0.58 & -0.04 & -3.27 & -0.85 & -0.04 & -1.74 & -4.07 & 0.81 \\
\hline chlorobenzene $\left(x_{1}\right)+n-C_{6}\left(x_{2}\right)$ & 0.5000 & 2.10 & 2.02 & 0.98 & -0.32 & -1.14 & 0.08 & -0.09 & -0.98 & -0.64 & -0.40 \\
\hline chlorobenzene $\left(x_{1}\right)+n-C_{8}\left(x_{2}\right)$ & 0.5000 & 2.29 & 2.34 & 0.70 & -0.62 & -1.63 & -0.05 & -0.11 & -1.13 & -0.94 & -0.57 \\
\hline chlorobenzene $\left(x_{1}\right)+n-C_{10}\left(x_{2}\right)$ & 0.5000 & 2.25 & 2.31 & 0.60 & -0.70 & -1.69 & -0.06 & -0.17 & -1.09 & -0.87 & -0.64 \\
\hline chlorobenzene $\left(x_{1}\right)+n-C_{12}\left(x_{2}\right)$ & 0.5000 & 1.63 & 1.87 & -0.15 & -0.86 & -1.81 & -0.24 & -0.12 & -0.88 & -1.06 & -0.62 \\
\hline chlorobenzene $\left(x_{1}\right)+n-C_{14}\left(x_{2}\right)$ & 0.5000 & 1.87 & 2.15 & -0.22 & -0.95 & -2.12 & -0.29 & -0.12 & -1.03 & -1.33 & -0.66 \\
\hline chlorobenzene $\left(x_{1}\right)+n-C_{16}\left(x_{2}\right)$ & 0.5000 & 1.48 & 1.85 & -0.63 & -1.00 & -2.14 & -0.38 & -0.08 & -0.90 & -1.44 & -0.62 \\
\hline chlorobenzene $\left(x_{1}\right)+b r-C_{6}\left(x_{2}\right)$ & 0.5000 & 1.43 & 1.61 & 0.24 & -0.24 & -1.21 & -0.18 & -0.13 & -0.75 & -1.01 & -0.06 \\
\hline chlorobenzene $\left(x_{1}\right)+b r-C_{8}\left(x_{2}\right)$ & 0.5000 & 1.45 & 1.62 & 0.27 & -0.27 & -1.19 & -0.18 & 0.02 & -0.83 & -1.12 & -0.09 \\
\hline chlorobenzene $\left(x_{1}\right)+b r-C_{12}\left(x_{2}\right)$ & 0.5000 & 0.75 & 0.74 & 0.38 & -0.05 & -0.37 & 0.01 & 0.03 & -0.39 & -0.34 & -0.06 \\
\hline chlorobenzene $\left(\mathrm{x}_{1}\right)+\mathrm{br}-\mathrm{C}_{16}\left(\mathrm{x}_{2}\right)$ & 0.5000 & 0.51 & 0.43 & 0.50 & 0.05 & -0.01 & 0.08 & 0.06 & -0.25 & -0.05 & -0.02 \\
\hline 1-chloronaphthalene $\left(x_{1}\right)+n-C_{8}\left(x_{2}\right)$ & 0.5000 & 3.00 & 2.82 & 2.12 & 0.56 & -0.91 & 0.18 & -0.06 & -1.41 & -1.23 & 0.38 \\
\hline 1-chloronaphthalene $\left(x_{1}\right)+n-C_{10}\left(x_{2}\right)$ & 0.5000 & 2.90 & 2.59 & 2.42 & 0.63 & -0.50 & 0.32 & -0.08 & -1.28 & -0.74 & 0.31 \\
\hline 1-chloronaphthalene $\left(x_{1}\right)+n-C_{12}\left(x_{2}\right)$ & 0.5000 & 2.74 & 2.44 & 2.26 & 0.51 & -0.49 & 0.30 & -0.09 & -1.20 & -0.61 & 0.21 \\
\hline 1-chloronaphthalene $\left(\mathrm{x}_{1}\right)+\mathrm{n}-\mathrm{C}_{14}\left(\mathrm{x}_{2}\right)$ & 0.5000 & 2.42 & 2.18 & 1.91 & 0.35 & -0.52 & 0.25 & -0.08 & -1.07 & -0.55 & 0.11 \\
\hline 1-chloronaphthalene $\left(x_{1}\right)+n-C_{16}\left(x_{2}\right)$ & 0.5000 & 1.99 & 1.72 & 1.67 & 0.15 & -0.33 & 0.27 & -0.07 & -0.84 & -0.13 & -0.13 \\
\hline 1-chloronaphthalene $\left(x_{1}\right)+b r-C_{6}\left(x_{2}\right)$ & 0.5000 & 3.95 & 4.52 & 0.86 & 0.28 & -3.22 & -0.59 & -0.25 & -2.21 & -3.86 & 0.87 \\
\hline 1-chloronaphthalene $\left(\mathrm{x}_{1}\right)+\mathrm{br}-\mathrm{C}_{8}\left(\mathrm{x}_{2}\right)$ & 0.5000 & 2.90 & 3.07 & 1.14 & 0.19 & -1.81 & -0.18 & -0.08 & -1.53 & -2.10 & 0.36 \\
\hline 1-chloronaphthalene $\left(x_{1}\right)+b r-C_{12}\left(x_{2}\right)$ & 0.5000 & 2.35 & 2.48 & 0.82 & 0.02 & -1.57 & -0.13 & -0.13 & -1.20 & -1.59 & 0.15 \\
\hline 1-chloronaphthalene $\left(\mathrm{x}_{1}\right)+\mathrm{br}-\mathrm{C}_{16}\left(\mathrm{x}_{2}\right)$ & 0.5000 & 2.38 & 2.52 & 0.73 & -0.07 & -1.69 & -0.15 & -0.12 & -1.22 & -1.64 & 0.07 \\
\hline
\end{tabular}

In the present work a number of useful and important properties of binary liquid mixtures have been theoretically estimated on the basis of Flory's statistical theory. The estimated properties are the thermal expansion coefficient, isothermal compressibility, adiabatic compressibility, heat capacity at constant pressure, internal pressure, density, heat capacity ratio, ultrasonic velocity, Gruneisen parameter and heat capacity at constant volume. Ultrasonic velocity is also calculated for pure components using Flory theory. Theoretically obtained values on the basis of a refined version of Flory theory developed by Pandey et al. (Pandey \& Sanguri, 2008; Pandey \& Sanguri, 2001; Pandey \& Sanguri, 2002; Pandey \& Sanguri, 2003; Dey \& Pandey et al, 2006), are compared with the experimental findings. It is clear from Table 2, that in general there is a good agreement between theoretical and experimental values of ultrasonic velocity of pure components. The maximum percentage deviation is $-16.63 \%$ for br- $\mathrm{C}_{6}$. In general the order of percentage deviation is about 3 to 5 percent. One of the reasons for slightly higher percentage deviation in ' $u$ ' is that we are using Auerbach empirical equation for the calculation of ultrasonic velocity for pure components. A close observation of Table 3 shows that the agreement between the experimental and theoretical values of all the estimated properties for all the systems is excellent. The maximum percentage deviation of each property is summarized below:

\begin{tabular}{|c|c|c|c|c|c|c|c|c|c|}
\hline$\beta_{T}$ & $\beta_{\mathrm{S}}$ & $\alpha$ & $\mathrm{C}_{\mathrm{p}}$ & $\mathrm{P}_{\mathrm{i}}$ & $\gamma$ & $\rho$ & $\mathrm{u}$ & $\Gamma$ & $\mathrm{C}_{\mathrm{V}}$ \\
\hline 4.91 & 5.84 & 2.42 & 1.79 & 4.41 & 0.98 & 0.46 & 2.97 & 5.11 & 1.30 \\
\hline
\end{tabular}

Table 4 enlists the percentage deviations of different properties (viz. $\beta_{\mathrm{s}}, \mathrm{C}_{\mathrm{p},}, \mathrm{P}_{\mathrm{i}}, \gamma, \Gamma \& \mathrm{C}_{\mathrm{v}}$ ) of binary mixtures calculated with the help of hole theory. The percentage deviation in the values of $\beta_{\mathrm{T}} \alpha$ and $u$ for the above 
Table 4. Percentage deviation of different properties of binary mixtures calculated through Hole theory

\begin{tabular}{|c|c|c|c|c|c|c|c|}
\hline \multirow[t]{2}{*}{ Components } & \multicolumn{5}{|c|}{ \% Deviation } & \multirow[b]{2}{*}{$\% \Gamma$} & \multirow[b]{2}{*}{$\% C_{v}$} \\
\hline & $\mathrm{x}_{1}$ & $\% \beta_{s}$ & $\% C_{p}$ & $\% \mathrm{P}_{\mathrm{i}}$ & $\% \gamma$ & & \\
\hline 1-chloronaphthalene $\left(\mathrm{x}_{1}\right)+\mathrm{n}-\mathrm{C}_{6}\left(\mathrm{x}_{2}\right)$ & 0.5000 & -2.57 & 43.75 & 16.32 & -13.06 & -68.13 & 50.25 \\
\hline 1-chloronaphthalene $\left(\mathrm{x}_{1}\right)+\mathrm{n}-\mathrm{C}_{8}\left(\mathrm{x}_{2}\right)$ & 0.5000 & 0.05 & -12.88 & -2.28 & 2.28 & 11.52 & -15.52 \\
\hline 1-chloronaphthalene $\left(x_{1}\right)+n-C_{10}\left(x_{2}\right)$ & 0.5000 & 1.08 & 5.01 & -0.91 & -1.88 & -8.15 & 6.77 \\
\hline 1-chloronaphthalene $\left(x_{1}\right)+n-C_{12}\left(x_{2}\right)$ & 0.5000 & 1.53 & 16.36 & 2.01 & -4.11 & -21.86 & 19.66 \\
\hline 1-chloronaphthalene $\left(x_{1}\right)+n-C_{14}\left(x_{2}\right)$ & 0.5000 & 1.50 & 20.00 & 0.58 & -6.59 & -32.35 & 24.95 \\
\hline 1-chloronaphthalene $\left(x_{1}\right)+n-C_{16}\left(x_{2}\right)$ & 0.5000 & 1.13 & 18.45 & -0.05 & -6.67 & -30.77 & 23.54 \\
\hline 1-chloronaphthalene $\left(\mathrm{x}_{1}\right)+\mathrm{br}-\mathrm{C}_{6}\left(\mathrm{x}_{2}\right)$ & 0.5000 & -2.49 & 37.67 & 13.26 & -9.50 & -52.01 & 43.08 \\
\hline 1-chloronaphthalene $\left(\mathrm{x}_{1}\right)+\mathrm{br}-\mathrm{C}_{8}\left(\mathrm{x}_{2}\right)$ & 0.5000 & -0.01 & -35.35 & -5.77 & 4.73 & 25.61 & -42.06 \\
\hline 1-chloronaphthalene $\left(\mathrm{x}_{1}\right)+\mathrm{br}-\mathrm{C}_{12}\left(\mathrm{x}_{2}\right)$ & 0.5000 & 2.19 & 8.58 & 3.14 & -0.06 & -5.87 & 8.63 \\
\hline 1-chloronaphthalene $\left(\mathrm{x}_{1}\right)+\mathrm{br}-\mathrm{C}_{16}\left(\mathrm{x}_{2}\right)$ & 0.5000 & 2.76 & -5.73 & -1.04 & 1.42 & 5.91 & -7.25 \\
\hline chlorobenzene $\left(x_{1}\right)+n-C_{6}\left(x_{2}\right)$ & 0.5000 & 3.30 & -12.48 & -9.59 & -1.53 & 1.07 & -10.78 \\
\hline chlorobenzene $\left(\mathrm{x}_{1}\right)+\mathrm{n}-\mathrm{C}_{8}\left(\mathrm{x}_{2}\right)$ & 0.5000 & 4.06 & 16.79 & -1.80 & -9.57 & -34.05 & 24.06 \\
\hline chlorobenzene $\left(x_{1}\right)+n-C_{10}\left(x_{2}\right)$ & 0.5000 & 3.57 & 35.75 & 6.60 & -15.15 & -67.41 & 44.21 \\
\hline chlorobenzene $\left(\mathrm{x}_{1}\right)+\mathrm{n}-\mathrm{C}_{12}\left(\mathrm{x}_{2}\right)$ & 0.5000 & 2.44 & 56.40 & 21.12 & -19.17 & -115.63 & 63.42 \\
\hline chlorobenzene $\left(x_{1}\right)+n-C_{14}\left(x_{2}\right)$ & 0.5000 & 13.91 & 58.33 & 12.24 & -25.64 & -164.63 & 66.83 \\
\hline chlorobenzene $\left(\mathrm{x}_{1}\right)+\mathrm{n}-\mathrm{C}_{16}\left(\mathrm{x}_{2}\right)$ & 0.5000 & 0.71 & 58.99 & 21.08 & -24.56 & -139.71 & 67.08 \\
\hline chlorobenzene $\left(x_{1}\right)+b r-C_{6}\left(x_{2}\right)$ & 0.5000 & 3.06 & 1.92 & 7.84 & 6.04 & 11.71 & -4.38 \\
\hline chlorobenzene $\left(\mathrm{x}_{1}\right)+\mathrm{br}-\mathrm{C}_{8}\left(\mathrm{x}_{2}\right)$ & 0.5000 & 4.68 & 42.01 & 18.47 & -3.79 & -45.90 & 44.12 \\
\hline chlorobenzene $\left(x_{1}\right)+b r-C_{12}\left(x_{2}\right)$ & 0.5000 & 1.24 & 49.92 & 18.54 & -12.57 & -83.11 & 55.51 \\
\hline chlorobenzene $\left(x_{1}\right)+b r-C_{16}\left(x_{2}\right)$ & 0.5000 & 1.70 & -4.20 & -2.08 & 0.45 & 2.47 & -4.67 \\
\hline benzene $\left(x_{1}\right)+n-C_{6}\left(x_{2}\right)$ & 0.5000 & 4.69 & -49.97 & -1.34 & 12.73 & 41.03 & -71.85 \\
\hline benzene $\left(x_{1}\right)+n-C_{8}\left(x_{2}\right)$ & 0.5000 & 4.72 & -34.78 & -9.54 & 5.57 & 23.25 & -42.73 \\
\hline benzene $\left(x_{1}\right)+n-C_{10}\left(x_{2}\right)$ & 0.5000 & 3.85 & -13.03 & -10.29 & -1.34 & 1.12 & -11.53 \\
\hline benzene $\left(x_{1}\right)+n-C_{12}\left(x_{2}\right)$ & 0.5000 & 2.86 & 30.82 & 5.07 & -11.67 & -53.24 & 38.05 \\
\hline benzene $\left(x_{1}\right)+n-C_{14(}\left(x_{2}\right)$ & 0.5000 & -1.87 & 22.26 & 3.31 & -11.98 & -39.26 & 30.57 \\
\hline benzene $\left.\left(x_{1}\right)+n-C_{16(} x_{2}\right)$ & 0.5000 & 1.32 & 35.76 & 3.63 & -18.74 & -78.12 & 45.90 \\
\hline toluene $\left(x_{1}\right)+n-C_{6}\left(x_{2}\right)$ & 0.5000 & 3.38 & -47.36 & -10.18 & 7.74 & 31.02 & -59.72 \\
\hline toluene $\left(x_{1}\right)+n-C_{8}\left(x_{2}\right)$ & 0.5000 & 4.93 & 9.64 & -1.90 & -3.93 & -17.20 & 13.06 \\
\hline toluene $\left(\mathrm{x}_{1}\right)+\mathrm{n}-\mathrm{C}_{10}\left(\mathrm{x}_{2}\right)$ & 0.5000 & 3.77 & 25.33 & 1.88 & -10.72 & -45.49 & 32.56 \\
\hline toluene $\left(\mathrm{x}_{1}\right)+\mathrm{n}-\mathrm{C}_{12}\left(\mathrm{x}_{2}\right)$ & 0.5000 & 3.41 & 12.41 & -3.73 & -7.11 & -26.84 & 18.22 \\
\hline toluene $\left(\mathrm{x}_{1}\right)+\mathrm{n}-\mathrm{C}_{14}\left(\mathrm{x}_{2}\right)$ & 0.5000 & -0.15 & 29.88 & 8.87 & -8.21 & -40.64 & 35.20 \\
\hline toluene $\left(\mathrm{x}_{1}\right)+\mathrm{n}-\mathrm{C}_{16}\left(\mathrm{x}_{2}\right)$ & 0.5000 & 1.76 & 41.03 & 10.26 & -13.18 & -72.26 & 47.90 \\
\hline$p$-xylene $\left(x_{1}\right)+n-C_{6}\left(x_{2}\right)$ & 0.5000 & -0.49 & -38.56 & -5.77 & 6.72 & 28.79 & -48.53 \\
\hline $\mathrm{p}$-xylene $\left(\mathrm{x}_{1}\right)+\mathrm{n}-\mathrm{C}_{16}\left(\mathrm{x}_{2}\right)$ & 0.5000 & -1.08 & 48.03 & 14.49 & -17.31 & -93.01 & 55.70 \\
\hline
\end{tabular}


Table 5. Percentage deviation of different properties of pure components using Hard sphere equation of states.

\begin{tabular}{|c|c|c|c|c|c|c|c|c|c|c|c|c|c|c|}
\hline Component & $\begin{array}{l}\% \beta_{\top} \\
(\mathrm{CS})\end{array}$ & $\begin{array}{c}\% \beta_{\mathrm{T}} \\
(\mathrm{TL})\end{array}$ & $\begin{array}{c}\% \beta_{T} \\
(\mathrm{~T})\end{array}$ & $\begin{array}{c}\% \beta_{\mathrm{T}} \\
(\mathrm{G}) \\
\end{array}$ & $\begin{array}{l}\% \beta_{\top} \\
(\mathrm{H}-\mathrm{R})\end{array}$ & $\begin{array}{c}\% \beta_{\top} \\
(\mathrm{ST})\end{array}$ & $\begin{array}{c}\% \beta_{\mathrm{T}} \\
(\mathrm{H}) \\
\end{array}$ & $\begin{array}{l}\% \alpha \\
\text { (CS) }\end{array}$ & $\begin{array}{l}\% \alpha \\
(\mathrm{TL})\end{array}$ & $\begin{array}{c}\% \alpha \\
(\mathrm{T})\end{array}$ & $\begin{array}{c}\% \alpha \\
(\mathrm{G})\end{array}$ & $\begin{array}{l}\% \alpha \\
(\mathrm{H}-\mathrm{R})\end{array}$ & $\begin{array}{l}\% \alpha \\
\text { (ST) }\end{array}$ & $\begin{array}{c}\% \alpha \\
(\mathrm{H})\end{array}$ \\
\hline benzene & 0.92 & 10.00 & -24.14 & 24.37 & -27.86 & -314.51 & -296.54 & 29.07 & 31.69 & 21.83 & 36.61 & 16.77 & -3.05 & -1.14 \\
\hline toluene & -15.59 & -4.60 & -46.34 & 12.89 & -52.44 & -395.72 & -373.63 & 22.31 & 25.25 & 14.09 & 30.83 & 7.80 & -13.03 & -10.90 \\
\hline$p$-xylene & -26.66 & -13.88 & -63.33 & 6.66 & -73.78 & -466.51 & -440.11 & 20.46 & 23.57 & 11.54 & 29.62 & 3.70 & -15.96 & -13.72 \\
\hline$n-C_{6}$ & 5.35 & 2.34 & -29.41 & 15.85 & -29.57 & -313.56 & -297.11 & 32.95 & 35.26 & 26.83 & 39.42 & 23.69 & 3.03 & 4.74 \\
\hline$n-C_{8}$ & -32.50 & -20.18 & -66.66 & -0.66 & -72.38 & -459.56 & -435.04 & 26.19 & 28.94 & 18.55 & 34.13 & 13.02 & -7.30 & -5.30 \\
\hline$n-C_{10}$ & -53.36 & -37.84 & -97.96 & -12.88 & -110.88 & -587.47 & -555.36 & 22.31 & 25.35 & 13.56 & 31.28 & 5.83 & -13.28 & -11.09 \\
\hline $\mathrm{n}-\mathrm{C}_{12}$ & -69.11 & -50.85 & -123.15 & -21.17 & -144.40 & -695.50 & -656.49 & 21.05 & 24.25 & 11.56 & 30.66 & 1.93 & -15.35 & -13.08 \\
\hline$n-C_{14}$ & -82.31 & -61.53 & -145.47 & -27.44 & -176.32 & -794.71 & -749.02 & 20.39 & 23.71 & 10.29 & 30.52 & -1.17 & -16.49 & -14.16 \\
\hline$n-C_{16}$ & -90.90 & -68.08 & -162.07 & -30.34 & -203.35 & -874.34 & -822.77 & 20.89 & 24.27 & 10.36 & 31.36 & -2.78 & -15.89 & -13.54 \\
\hline $\mathrm{br}-\mathrm{C}_{6}$ & -2.28 & 5.58 & -22.71 & 17.66 & -21.43 & -283.07 & -268.53 & 34.28 & 36.46 & 28.64 & 40.30 & 26.28 & 5.22 & 6.85 \\
\hline $\mathrm{br}-\mathrm{C}_{8}$ & -30.38 & -19.01 & -61.20 & -1.16 & -63.80 & -428.02 & -405.97 & 25.25 & 27.93 & 17.98 & 32.87 & 13.51 & -8.40 & -6.43 \\
\hline $\mathrm{br}-\mathrm{C}_{12}$ & --- & --- & --- & --- & --- & --- & --- & --- & --- & --- & --- & --- & --- & --- \\
\hline $\mathrm{br}-\mathrm{C}_{16}$ & --- & --- & --- & --- & --- & --- & --- & --- & --- & --- & --- & --- & --- & --- \\
\hline chlorobenzene & -17.15 & -5.35 & -50.99 & 13.61 & -60.55 & -423.38 & -399.01 & 17.37 & 20.60 & 8.12 & 26.88 & 0.02 & -20.45 & -18.14 \\
\hline $\begin{array}{l}\text { 1-chloro- } \\
\text { naphthalene }\end{array}$ & -65.79 & -46.40 & -125.55 & -14.45 & -157.65 & -730.95 & -687.68 & -3.45 & 0.92 & -16.93 & 9.99 & -33.03 & -51.48 & -48.42 \\
\hline
\end{tabular}

mentioned systems are presented in our earlier paper (Pandey \& Sanguri et al., 2007). All the calculated properties show the same trend as obtained experimentally. The maximum percentage deviations of the calculated values of $\beta_{\mathrm{s}}, \mathrm{P}_{\mathrm{i}}, \gamma$ is $13.91,21.12$ and 25.64 respectively. In general the percentage deviation is below $7 \%$. In case of $C_{P}$, $\mathrm{C}_{\mathrm{V}} \& \Gamma$ the percentage deviation is little higher.

In Table 5 percentage deviations in the values of $\beta_{\mathrm{T}}$ and $\alpha$ of pure components, calculated by seven different HSE are reported. It is clear from Table 5 that in case of $\beta_{\mathrm{T}}$, only Guggenheim model gives some satisfactory results. The rest 6 models (viz. Thiele-Lebowitz, Thiele, CarnahanStarling, Scaled-particle theory, Henderson and HooverRee model) are not found suitable for the calculation of $\beta_{\mathrm{T}}$. In very few cases Thiele-Lebowitz and Carnahan-Starling models give satisfactory results. In the case of $\alpha$, Henderson and Scaled-particle theory models give satisfactory results followed by other models Hoover-Ree model, Thiele, Carnahan- Starling, Thiele-Lebowitz \& Guggenheim.

Table 6 enlists percentage deviations in the theoretical values of $\beta_{\mathrm{T}}$ and $\alpha$ of equimolar binary mixtures by seven different HSE. It is clear from Table 6 that similar types of results are obtained in the case of isothermal compressibility of binary mixtures as in the case of pure components. For thermal expansivity, different types of trends are observed. For the systems (benzene + alkanes) and (toluene + alkanes) Henderson and Scaled-particle theory models give satisfactory results. Other models show higher deviations. For the system (chlorobenzene + alkanes) Hoover-Ree model gives good agreement followed by Thiele \& Henderson models which gives slightly higher deviations. Rest models give percentage deviations between
20-30\%. In the case of system (1-chloronaphthalene + alkanes) Thiele model gives very good agreement, while Hoover-Ree and Carnahan-Starling models give satisfactory agreement.

\section{Conclusion}

The results obtained by applying FST, HSE and HT to the pure liquids and 34 equimolar binary mixtures at $298.15 \mathrm{~K}$ are remarkable. Out of the ten properties of binary mixtures calculated by FST, the two most important properties viz. density and ultrasonic velocity give excellent agreement with the experimental findings collected from literature as the APD values are found to be in the order of $1 \%$ (in case of density) and below $2 \%$ (in case of ultrasonic velocity). In case of density, the maximum APD is found to be $0.46 \%$. The APD values for the rest of the eight properties are below $6 \%$ which is excellent.

In general, the percentage deviation is below $7 \%$ for $\beta_{\mathrm{s}}$, $\mathrm{P}_{\mathrm{i}}$, and $\gamma$ in case of Hole theory, whereas for $\mathrm{C}_{\mathrm{P}}, \mathrm{C}_{\mathrm{V}} \& \Gamma$ the percentage deviation is little higher. For $\beta_{T}$ out of the seven HSE, only Guggenheim model gives some satisfactory results, other models are not found to be suitable for the calculation of $\beta_{\mathrm{T}}$. In a few cases, Thiele-Lebowitz and Carnahan-Starling model gives satisfactory results. For thermal expansivity different types of trends are observed but in most of the cases Henderson model and Scaledparticle theory give satisfactory results. The above results have led to the conclusion that FST is superior in all respects to HSE and HT.

Since theoretically obtained values of density and ultrasonic velocity by FST have APD values below $1 \%$ and $2 \%$ respectively in the present work, as well as in our 


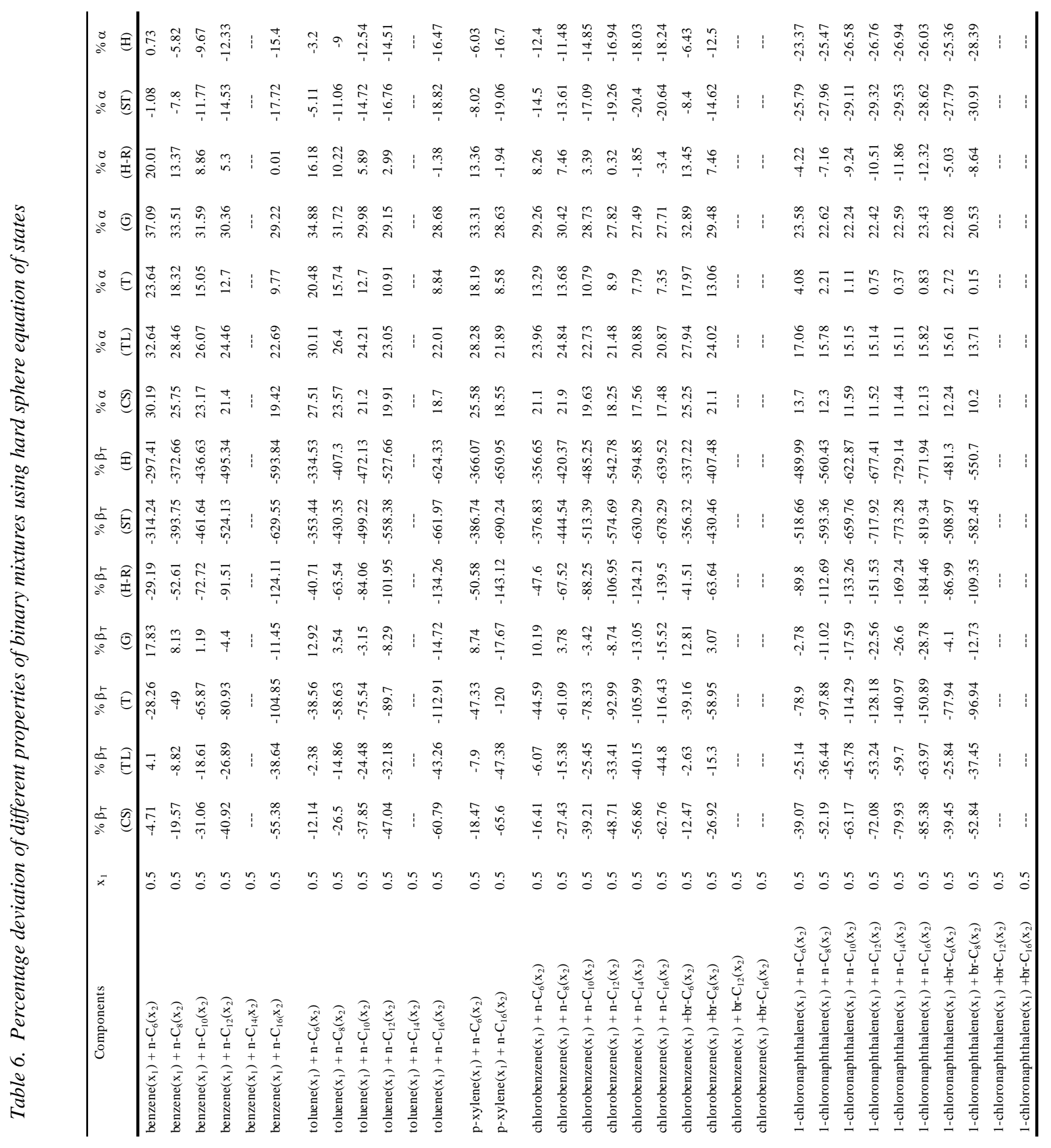


earlier obtained results (Pandey \& Sanguri, 2008; Pandey \& Sanguri, 2001), therefore, FST can be used as a tool to verify and cross-check the experimental data as there can be numerous errors viz. manual errors, instrumental error etc. at the time of recording data.

\section{References}

Abe, A. \& Flory, P. J. (1965). The Thermodynamic Properties of Mixtures of Small, Nonpolar Molecules. $J$. Am. Chem. Soc., 87, 1838-1846.

Acosta, J., Acre, A., Rodil, E., \& Soto, A.(2001). Densities, Speeds of Sound, Refractive indices and the corresponding changes of Mixing at $25^{\circ} \mathrm{C}$ and atmospheric pressure for systems composed by Ethyl acetate, Hexane and Acetone. J. Chem. Eng. Data, 46(5), 1176-1180.

Acree (Jr), W. E. (1984). Thermodynamic Properties of Nonelectrolyte Solutions, I N C London Ltd: Academic Press.

Ali, A. \& Nain, A. K. (2000). Study of molecular interactions in non-aqueous binary liquid mixtures through ultrasonic measurements. J. Pure Appl. Ultrason., 22, 10-15.

Ali, A., \& Nain, A.K. (2002).Ultrsonic \& Volumetric Study of Binary Mixtures of Benzyl Alcohol with Amides. Bull.Chem. Soc.Japan, 75(4), 681-687.

Ali, A. \& Nain, A. K. (2002). Ultrasonic study of molecular interaction in binary liquid mixtures at $30^{\circ} \mathrm{C}$. Pramana 58(4), 695-701.

Ali, A., Nain, A. K., Sharma, V. K. \& Ahmad, S. (2000). Acoustical studies of molecular interaction in binary liquid mixtures at $35^{\circ}$ C. J. Acoust. Soc. India, 28, 283288.

Ali, A., Tiwari, K., Nain, A.K., \& Chakravortty, V. (2000). Ultrasonic study of molecular interaction in ternary mixtures of dimethyl sulphoxides (1) + carbon tetrachloride (2) + aromatic hydrocarbons (3) at 308.15K. Indian J. Phys., 74B, 351-355.

Arce. A., Arce, A. Jr., Ageitos, J.M., Rodil, E., Rodríguez,O., \& Soto, A. (2000). . Physical and equilibrium properties of diisopropyl ether+isopropyl alcohol+water system. Fluid Phase Equilibria, 170, 113126.

Baluja, S. (2002). Ultrasonic studies of resorcinol in protic $\&$ aprotic solvents at $40^{\circ}$. J. Indian Chem. Soc., 79, 142144.

Bloomfeld, V. A. \& Dewan, R. K. (1971). Viscosity of liquid mixtures. J Phys. Chem., 75, 3113-3119.

Canosa, J., Rodrı'guez,A., \& Tojo, J. (2001). Liquid-Liquid Equilibrium and Physical Properties of the Ternary
Mixture (Dimethyl Carbonate + Methanol + Cyclohexane) at 298.15 K. J. Chem. Eng. Data, 46, 846-850.

Carnahan, N. F., Starling, K. E. (1969). Equation of State for Nonattracting Rigid Spheres. J. Chem. Phys., 51, 635-636.

Costas, M., Tra, H. V., Patterson, D., Alonso, M. C., Tardajos, G. \& Aicart, E. (1988). Liquid structure and second-order mixing functions for 1-chloronaphthalene with linear and branched alkanes. J. Chem. Soc. Faraday Trans 1, 84, 1603-1616.

CRC Handbook of Chem. \& Phys., (1997-98). (78 Edition) New York: CRC Press, Boca Raton.

Dewan, R. K., Bloomfeld, V. A. \& Berget, P. B. (1971). Intrinsic viscosity of short-chain normal alkanes. $J$. Phys. Chem., 75, 3120-3124.

Dey, R., Soni, N. K., Mishra, R. K., Sanguri, V. \& Pandey, J. D. (2006). Thermal conductivity of ternary liquid mixtures: Application of modified Flory statistical theory and uniform gaseous model. J. Mol. Liq., 124, 102-105.

Dhasi, V., Wadhawani, R., Akhtar, Y., Pandey, J.D. \& Vyas,V. (1998). Ultrasonic and Thermodynamic Studies of Ternary Solutions. Acustica-Acta Acustica, 84, 976-979.

Dominguez, A., Tardajos, G., Aicart, E., Casas, S. P., Trejo, L. M., Costas, M., Patterson, D. \& Tra, H. V. (1993). Van der Waals liquids, Flory theory and mixing functions for chlorobenzene with linear and branched alkanes. J. Chem. Soc. Faraday Trans 1, 89, 89-94.

Eyring, H. \& John, M. S. (1969). Significant Liquid Structures, New York: John Wiley \& Sons Inc.

Flory, P. J. (1965). Statistical Thermodynamics of Liquid Mixtures. J. Am. Chem. Soc., 87, 1833-1838.

Flory, P. J., Orwoll, R. A. \& Vrij, A. (1964). Statistical Thermodynamics of Chain Molecule Liquids. I. An Equation of State for Normal Paraffin Hydrocarbons. $J$. Am. Chem. Soc., 86, 3507-3514.

Frisch HL, (1964). The equation of state of the classical hard sphere fluids. Adv Chem. Phys, 6, 229-289.

Hoover, W.G. \& Ree, F.H, (1964). Fifth and Sixth Virial Coefficients for Hard Spheres and Hard Disks. J. Chem. Phys., 40, 939-950.

Jain, D.V.S., Alastair, M. N., \& Pethrick, R.A. (1974). Adiabatic compressibility of binary liquid mixtures. $J$. Chem. Soc., Faraday Trans 1,70, 1292- 1298.

Jakobson, B. (1951). Intermolecular Free Lengths in Liquids in Relation to Compressibility, Surface Tension and Viscosity. Acta Chem. Scad.,5, 1214-1216. 
Kaulgud, M.V. \& Patil, K. J. (1972). Sound propagation in some mono and disubstituted derivatives of benzene. Acustica, 26, 292-296.

Lebowitz, J.L., Frisch, H. L. \& Helfand, E. (1961). Theory of the Two- and One-Dimensional Rigid Sphere Fluids. J. Chem. Phys., 34, 1037-1042.

Lebowitz, J. L., Helfand, E. \& Praestgaard, E. (1965). Scaled Particle Theory of Fluid Mixtures. J. Chem. Phys., 43, 774-778.

Mosteriro, L., Mascato, E., deConinges, B.E., Iglesias, T.P., \& Legido, J.L. (2001). Density, speed of sound, refractive index and dielectric permittivity of (diethyl carbonate + n-decane) at several temperatures. J. Chem. Thermodyn., 33, 787-801.

Murrell, J. N. \& Jenkins, A. D. (1982). Properties of Liquids \& Solutions, New York: John Willy \& Sons.

Nomoto, O. (1958). Empirical Formula for Sound Velocity in Liquid Mixtures. J. Phys. Soc. Jpn. 13, 1528-1532.

Pandey, J. D. \& Alec David, D. M. (1981). Viscosities of binary molten nitrate mixtures. J. Phys. Chem., 85, 3151-3152.

Pandey, J. D., Dey, R \& Dwivedi, D. K. (1999). Ultrasonic velocity of binary systems at elevated pressures. Pramana-j phys, 52, 187-193.

Pandey, J. D., Dubey, G. P., Dey, R. \& Dubey, S. N. (1997). Temperature and Pressure Dependence of Thermoacoustical Parameters of Liquid Argon and Xenon. Acustica, 83, 90-92.

Pandey, J. D., Dubey, G. P., Shukla, B. P. \& Dubey, S.N. (1994). A Comparative Study of Isothermal Compressibility from Ultrasonic Velocity and Flory's Statistical Theory for Various Binary Mixtures. Acustica, 80, 92-96.

Pandey, J. D. \& Sanguri, V. (2001). Prediction of density of liquid mixture using Flory Statistical Theory. $J$. Chem. Research (S), 344-345.

Pandey J. D. \& Sanguri, V. (2008). Theoretical estimations of thermodynamic properties of liquid mixtures by Flory's statistical theory. Phys. Chem. Liq., 46, 417432.

Pandey, J. D., Sanguri, V. \& Bhatt, B. D. (2003). Application of Flory's statistical theory for the estimation of internal pressure of the liquid mixtures. $J$. Chem. Research(S), 430-432.

Pandey, J. D., Sanguri, V. \& Dwivedi, D. K. (2008). Thermodynamic properties of pure liquids within a generalized version of the hole theory. Phys. Chem. Liq., 46, 162-171.

Pandey, J. D., Sanguri, V., Dwivedi, D. K. \& Tiwari K. K. (2007). Computation of isothermal compressibility, thermal expansivity and ultrasonic velocity of binary liquid mixtures using hole theory. J. Mol. Liq., 135, 6571.

Pandey, J. D., Srivastava, T., Chandra P., Dwivedi, D. K. \& Sanguri, V. (2010). Estimation of thermodynamic properties of multicomponent systems on the basis of generalized version of hole theory. J. Mol. Liq., 157, 158-161.

Pandey, J. D., Tripathi, S. B. \& Sanguri, V. (2002). Thermal Expansivity of multi-component liquid systems. J. Mol. Liq., 100/2, 153-161.

Pandey, J. D., Vyas, V., Jain, P., Dubey, G. P., Tripathi, N. \& Dey. R. (1999). Speed of sound, viscosity and refractive index of multicomponent systems: theoretical predictions from the properties of pure components. $J$. Mol. Liq. 81, 123-133.

Percus, J. K. \& Yevick, G.J. (1958). Analysis of Classical Statistical Mechanics by Means of Collective Coordinates. Phy. Rev, 110, 1-13.

Prausnitz, J. M., Lichtenthaler, R. N. \& de Azevedo, (1986). Molecular Thermodynamic of Fluid Phase Equilibria, Englewood Cliffs, N J: Printice-Hall Ind.

Reid, R. C., Prausnitz, J. M. \& Poling, B. E. (1987). The Properties of Gases and Liquids, New York: Mc-Graw Hill.

Rowlinson, J. S. \& Swinton F. L. (1982). Liquids \& Liquid Mixture, London: Butter worth scientific.

Sanguri, V., Dwivedi, D. K., Singh, N., Pandey, N. \& Pandey, J. D. (2008). Thermodynamic properties of multicomponent systems and hole theory. J. Mol. Liq., $141,1-7$.

Schaffs, W. (1939). Zur Bestimmung von Molekülradien organischer Flüssigkeiten aus Schallgeschwindigkeit und Dichte. Z. Phys., 114, 110-115.

Tardajos, G., Aicart, E., Costas, M. \& Patterson, D. (1986). Liquid structure and second-order mixing functions for benzene, toluene and $p$-xylene with n-alkanes J. Chem. Soc. Faraday Trans. 1, 82, 2977- 2988.

Thiele, E. J. (1963). Equation of State for Hard Spheres. J. Chem. Phys., 39, 474-478.

Van Dael, W. (1975). Thermodynamics properties and velocity of sound, London: Butterworth. 